\title{
High Human Antimicrobial Peptide LL-37 Level Predicts Lower Major Adverse Cardiovascular Events after an Acute ST-Segment Elevation Myocardial Infarction
}

\author{
Hanjun Zhao ${ }^{1}$, Zhaoxue Sheng ${ }^{1,2}$, Yu Tan ${ }^{1}$, Runzhen Chen ${ }^{1}$, Jinying Zhou ${ }^{1}$, Jiannan Li ${ }^{1}$, Qianyu Zhao ${ }^{3}$, \\ Ying Wang ${ }^{1}$, Xiaoxiao Zhao ${ }^{1}$, Yi Chen ${ }^{1}$, Peng Zhou ${ }^{1}$, Chen Liu ${ }^{1}$, Li Song ${ }^{1}$ and Hongbing Yan ${ }^{1,4}$
}

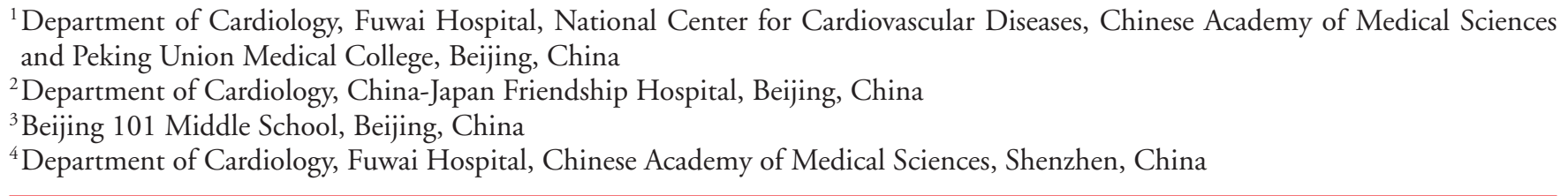

Aims: We previously associated acute ST-elevation myocardial infarction (STEMI) with decreased plasma LL-37 levels. Therefore, this study investigated whether plasma LL-37 levels could predict ischemic cardiovascular events in patients after STEMI.

Methods: We prospectively collected peripheral plasma samples and clinical and laboratory data from consecutive patients who presented with STEMI and underwent primary percutaneous coronary intervention at Fuwai Hospital between April and November 2017. Enzyme-linked immunosorbent assay measured plasma LL-37 levels, and we followed the patients for 3 years. Major adverse cardiovascular events (MACEs) were a composite of all-cause mortality, reinfarction, unscheduled revascularization, or ischemic stroke.

Results: The study included 302 patients divided into high ( $\geq$ median) and low LL-37 level ( $<$ median) groups. The cumulative incidence of MACE $(29.1 \%$ vs. $12.6 \%, p=0.0003)$, all-cause death $(12.6 \%$ vs. $3.3 \%, p=0.003)$, reinfarction $(7.1 \%$ vs. $2.0 \%, p=0.04)$, and unscheduled revascularization $(13.0 \%$ vs. $5.4 \%, p=0.04)$ were higher in the low than those in the high LL-37 level group. Multivariable Cox regression analysis showed that higher LL-37 level independently predicted lower risks of MACE (hazard ratio [HR] 0.390; 95\% confidence interval [CI] 0.227-0.669; $p<0.001$ ), all-cause death (HR 0.324; 95\%CI 0.119-0.879; $p=0.027$ ), and unscheduled revascularization (HR 0.391; 95\%CI 0.171-0.907; $p=0.027$ ).

Conclusions: High basal plasma level of human LL-37 may predict lower 3-year risks of ischemic cardiovascular events in patients after STEMI.

Key words: LL-37, Acute myocardial infarction, All-cause death, Reinfarction, Revascularization

\section{Introduction}

Despite stringent modification of traditional risk factors, widespread use of primary percutaneous coronary intervention (PCI), and guideline-directed medical therapy, mortality and morbidity of acute myocardial infarction (AMI) remain high ${ }^{1)}$. Therefore, residual risk factors should be urgently identified to improve our understanding of the pathophysiology of atherosclerosis and AMI and to determine novel therapeutic targets. Atherosclerosis is an immuneinflammatory disease, and inflammation drives all phases of atherosclerosis, including initiation, progression, and thrombotic complications ${ }^{2-4}$. The antimicrobial peptide LL-37 is a positively charged, multifunctional, 37-residue host defense molecule

Address for correspondence: Hongbing Yan, Fuwai Hospital, No.167, Beilishi Road, Xicheng District, Beijing 100037, China E-mail: hbyanfuwai2018@163.com Received: August 16, 2021 Accepted for publication: October 12, 2021 


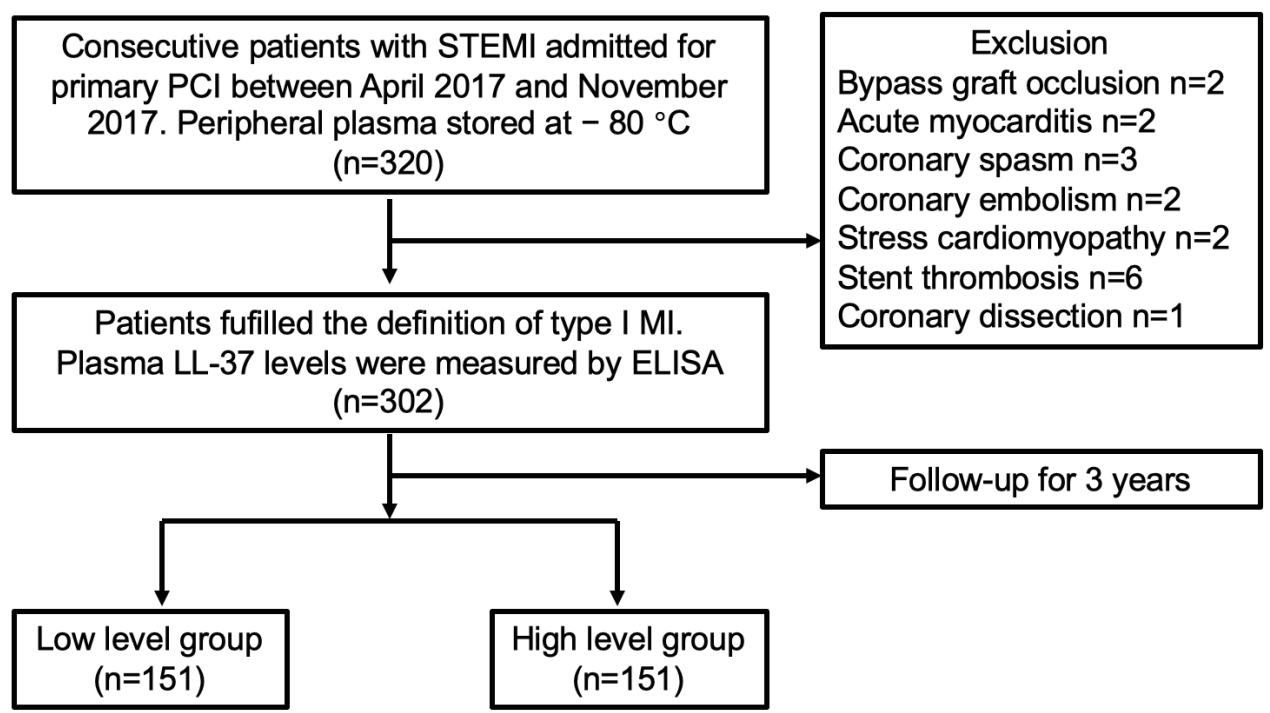

Fig. 1. Study flow chart

ACS: acute coronary syndrome; MI: myocardial infarction; STEMI: acute ST-elevation myocardial infarction; ELISA: enzyme-linked immunosorbent assay.

derived from the human cationic antimicrobial protein 18 (hCAP-18), which cleaved in neutrophils by proteinase- $3^{5)}$. LL-37 is the only cathelicidin in humans produced by various types of cells, including skin, epithelial, and innate immune system cells ${ }^{6}$. In addition to the broad spectrum of antimicrobial activity, LL-37 attracts innate immune cells to the site of infection and modulates immune reactivity and the balance between pro- and anti-inflammatory responses. It also induces wound healing and angiogenesis and modulates apoptosis ${ }^{7,8)}$.

Some experimental studies indicated LL-37 might have cardiovascular protective effects. A nitinol stent coated with LL-37 reduces in-stent stenosis in mouse carotid artery, suggesting that LL-37 may promote vascular healing after an interventional therapy ${ }^{9)}$. LL-37 inhibits platelet aggregation and thrombosis via the Src/PI3K/Akt pathway, suggesting its antiplatelet and antithrombotic actions ${ }^{10)}$. Previously, we reported that the basal level of plasma LL-37 was significantly lower in patients with acute ST-elevation myocardial infarction (STEMI) than those without coronary artery disease (CAD) and stable CAD, and its level seems to recover within $24 \mathrm{~h}$ after STEMI ${ }^{11)}$. However, the clinical association between LL-37 levels and cardiovascular events in patients after STEMI remains unknown. Therefore, we designed this study to explore LL-37's prognostic value for patients after STEMI.

\section{Methods}

Study Population

This prospective observational cohort study is part of the Chinese Academy of Medical Sciences Innovation program. We recruited consecutive patients aged $\geq 18$ years who presented with STEMI and underwent primary PCI at Fuwai Hospital between April and November 2017 (Fig. 1). The exclusion criteria were cardiogenic shock, end-stage renal disease, serious liver dysfunction, allergy to contrast media, contraindication to antithrombotic therapy, bypass graft occlusion, and diagnosis not fulfilling the definition of type I myocardial infarction. STEMI was defined as continuous chest discomfort lasting for $>30 \mathrm{~min}$, ST-segment elevation of $>0.1$ $\mathrm{mV}$ in at least two contiguous leads or new left bundle-branch block on an 18-lead electrocardiogram (ECG), and an elevated troponin I level ${ }^{12)}$. This study complied with the Declaration of Helsinki and was approved by the Ethics Committee of Fuwai Hospital. All participants provided their written informed consent.

Procedural Data and Culprit Lesion Identification

Coronary angiography (CAG) was performed via the transradial or transfemoral approach using a 6-F or $7-\mathrm{F}$ sheath. Blood samples were collected via the sheath before heparinization using vacutainer tubes containing ethylenediaminetetraacetic acid. Samples were maintained at $4{ }^{\circ} \mathrm{C}$, processed within $3 \mathrm{~h}$, and 
then stored at $-80^{\circ} \mathrm{C}$ until further analysis. CAG was performed after heparinization (3000 units), and heparin was added to 100 units/kg before PCI. All patients received standard-of-care therapy according to international guidelines during the follow-up ${ }^{12}$. The culprit vessel was determined primarily by CAG and corroborated with ECG information and regional-wall motion abnormalities from echocardiographic or ventriculographic assessments.

\section{Laboratory Testing}

Plasma LL-37 levels were measured using a ready-to-use solid-phase enzyme-linked immunosorbent assay (ELISA) based on the sandwich principle with commercially available kits, human LL-37 ELISA Kit (HK321), purchased from HyCult Biotechnology (Uden, The Netherlands). The assay was performed according to the manufacturer's protocol.

\section{Follow-up and Outcome Definitions}

The primary outcome was major adverse cardiovascular events (MACEs), defined as a composite of all-cause mortality, reinfarction, unscheduled revascularization, or ischemic stroke. Secondary outcomes included each component of MACEs. All-cause mortality included cardiac death, vascular death, and deaths due to non-cardiovascular causes. Unscheduled revascularization included any clinically driven repeat coronary revascularization (target and non-target vessels). Medical record reviews and follow-up telephone interviews or clinical visits were conducted at the 30-day, 3-month, 6-month, 1-year, and 3-year follow-up.

\section{Statistical Analysis}

Continuous variables are presented using mean \pm standard deviation if they follow the normal distribution. We compared them using Student's $t$-test. Otherwise, they are presented as medians (interquartile range) and compared using MannWhitney $U$ test. Categorical variables are presented as numbers (\%) and analyzed with chi-square tests or Fisher's exact tests as necessary. We used Pearson's and Spearman's correlation analyses to examine the associations between LL-37 levels and other clinical features and laboratory test metrics. Kaplan-Meier curve analysis compared events between two groups. Multivariable Cox regression assessed the association between LL-37 levels and various outcomes, followed by subgroup analysis across established clinical risk factors (e.g., age, gender, left ventricular ejection fraction [LVEF], hypertension, diabetes, levels of lowdensity lipoprotein cholesterol [LDL-C], total cholesterol, and triglycerides). Restricted cubic spline analysis was used to assess the dose-response association between LL-37 and primary outcome. We performed the analyses using Statistical Package for the Social Sciences 25 (IBM Corp., Armonk, NY, USA) and $R$ statistical software version 3.6.2 (www. R-project.org, $\mathrm{R}$ Foundation for Statistical Computing). A two-tailed $p$ value of $<0.05$ was considered statistically significant.

\section{Results}

\section{Baseline Characteristics}

The study enrolled 302 patients with STEMI, and all of them completed the follow-up (Fig. 1), with the mean age of 59.1 years, comprising $81.1 \%$ men, $59.1 \%$ with hypertension, and $28.1 \%$ with diabetes. The median LL-37 level was 30.4 (19.7-41.1) $\mu \mathrm{g} / \mathrm{L}$, and all patients were divided into two groups according to their LL-37 levels: high (LL-37 $\geq$ median) and low-level groups (LL-37<median). Tables 1 and 2 list comparisons of baseline clinical characteristics between the two groups. We observed no differences in age, sex, body mass index, and laboratory and coronary angiographic findings, except that triglyceride, total cholesterol (TC), and lowdensity lipoprotein cholesterol (LDL-C) levels were higher in the high than those in the low LL-37 group $(p=0.008, p=0.004$, and $p=0.019$, respectively). Conversely, thrombolysis in myocardial infarction (TIMI), Global Registry of Acute Coronary Events (GRACE) scores, and history of ischemic stroke were lower in the high than that in the low LL-37 group ( $p=0.018, p=0.002$, and $p=0.044$, respectively) (Table 1). No differences occurred in medical therapies at discharge between the two groups (Table 2).

Correlation Analysis of LL-37 and Clinical Data

Correlation analysis between LL-37 and clinical data showed that LL-37 levels were positively correlated with triglyceride $(\mathrm{r}=0.218, p<0.001)$, TC $(\mathrm{r}=0.154, p=0.008)$, and LDL-C $(\mathrm{r}=0.124$, $p=0.033)$ and negatively correlated with TIMI and GRACE scores $(\mathrm{r}=-0.209, p<0.001 ; \mathrm{r}=-0.16$, $p=0.005$, respectively) (Table 3 ).

Association between LL-37 and Clinical Outcomes During the 3-year follow-up, 63 patients $(20.9 \%)$ had MACEs, 24 (7.9\%) died, 13 (4.5\%) suffered from reinfarction, $26(9.1 \%)$ underwent unscheduled revascularization, and $14(4.9 \%)$ suffered from the ischemic stroke (Table 4). Kaplan-Meier curve analysis showed the cumulative incidence of MACE 
Table 1. Baseline Characteristics According to Binary LL37 Groups

\begin{tabular}{|c|c|c|c|c|}
\hline Variables & Total $(n=302)$ & \multicolumn{2}{|c|}{ LL37 } & $p$ value \\
\hline Age & $59.1 \pm 13.0$ & $60.2 \pm 13.9$ & $58.0 \pm 12.0$ & 0.141 \\
\hline Men & $245(81.1 \%)$ & $121(80.1 \%)$ & $124(82.1 \%)$ & 0.769 \\
\hline BMI $\left(\mathrm{kg} / \mathrm{m}^{2}\right)$ & $25.8 \pm 3.9$ & $25.5 \pm 4.3$ & $26.2 \pm 3.4$ & 0.137 \\
\hline Smoker & $194(64.2 \%)$ & $101(66.9 \%)$ & $93(61.6 \%)$ & 0.401 \\
\hline Hypertension & $178(59.1 \%)$ & $89(58.9 \%)$ & $89(59.3 \%)$ & 1.000 \\
\hline OMI & $39(12.9 \%)$ & $21(13.9 \%)$ & $18(11.9 \%)$ & 0.732 \\
\hline Ischemic stroke & $34(11.3 \%)$ & $23(15.2 \%)$ & $11(7.3 \%)$ & 0.044 \\
\hline Previous PCI & $52(17.2 \%)$ & $30(19.9 \%)$ & $22(14.6 \%)$ & 0.286 \\
\hline \multicolumn{5}{|l|}{ Laboratory test } \\
\hline Hemoglobin $(\mathrm{g} / \mathrm{L})$ & $145.8 \pm 19.3$ & $143.9 \pm 20.5$ & $147.7 \pm 17.8$ & 0.089 \\
\hline Platelets $\left(10^{6} / \mathrm{L}\right)$ & $229 \pm 93$ & $220 \pm 65$ & $236 \pm 113$ & 0.141 \\
\hline hsCRP (mg/L) & $5.36(1.93-10.94)$ & $4.81(1.54-11.01)$ & $5.94(2.39-10.92)$ & 0.361 \\
\hline $\operatorname{ESR}(\mathrm{mm} / \mathrm{h})$ & $7(3-16)$ & $7(3-18)$ & $7(3-14)$ & 0.656 \\
\hline $\mathrm{eGFR}\left(\mathrm{mL} / \mathrm{min} / 1.73 \mathrm{~m}^{2}\right)$ & $100.0 \pm 30.6$ & $98.58 \pm 33.08$ & $101.42 \pm 28.05$ & 0.422 \\
\hline Glycosylated hemoglobin (\%) & $6.4 \pm 1.4$ & $6.3 \pm 1.2$ & $6.5 \pm 1.5$ & 0.255 \\
\hline Triglyceride & $1.7 \pm 1.1$ & $1.5 \pm 0.9$ & $1.8 \pm 1.2$ & 0.008 \\
\hline $\mathrm{TC}$ & $4.3 \pm 1.1$ & $4.2 \pm 1.1$ & $4.5 \pm 1.0$ & 0.004 \\
\hline LDL-C & $2.7 \pm 0.9$ & $2.6 \pm 0.8$ & $2.8 \pm 0.9$ & 0.019 \\
\hline HDL-C & $1.1 \pm 0.3$ & $1.1 \pm 0.3$ & $1.1 \pm 0.3$ & 0.286 \\
\hline RCA & $117(38.7 \%)$ & $58(38.4 \%)$ & $59(39.1 \%)$ & \\
\hline LM & $3(1.0 \%)$ & $1(0.7 \%)$ & $2(1.3 \%)$ & \\
\hline Number of diseased arteries & & & & 0.620 \\
\hline Single & $78(25.8 \%)$ & $44(29.1 \%)$ & $34(22.5 \%)$ & \\
\hline Double & $97(32.1 \%)$ & $47(31.1 \%)$ & $50(33.1 \%)$ & \\
\hline Triple & $101(33.4 \%)$ & $48(31.8 \%)$ & $53(35.1 \%)$ & \\
\hline LM disease & $26(8.6 \%)$ & $12(7.9 \%)$ & $14(9.3 \%)$ & \\
\hline \multicolumn{5}{|l|}{ Clinical prognostic score } \\
\hline TIMI score & $3.8 \pm 2.3$ & $3.9 \pm 2.3$ & $3.3 \pm 2.0$ & 0.017 \\
\hline GRACE score & $102.1 \pm 31.0$ & $104.5 \pm 32.7$ & $95.1 \pm 26.9$ & 0.006 \\
\hline
\end{tabular}

BMI: body mass index; OMI: old myocardial infarction; PCI: percutaneous coronary intervention; hsCRP: high sensitive C-reactive protein; ESR: erythrocyte sedimentation rate; eGFR: estimated glomerular filtration rate; TC: total cholesterol; LDL-C: low-density lipoprotein cholesterol; HDL-C: high-density lipoprotein cholesterol; LVEDD: left ventricular end-diastolic dimension; LVEF: left ventricular ejection fraction; LAD: left anterior descending artery; LCX: left circumflex artery; RCA: right coronary artery; LM: left main; TIMI: thrombolysis in myocardial infarction; GRACE: global registry of acute coronary events. 
Table 2. Medication at Discharge

\begin{tabular}{lcc}
\hline Variables & \multicolumn{2}{c}{ LL-37 value } \\
\cline { 2 - 3 } & Low group $(n=151)$ & High group $(n=151)$ \\
\hline Antithrombotic therapy & & $0(0 \%)$ \\
$\quad$ Mono-antiplatelet therapy (MAPT) & $2(1.3 \%)$ & $148(98.0 \%)$ \\
Dual antiplatelet therapy (DAPT) & $138(91.4 \%)$ & $1(0.7 \%)$ \\
MAPT + anticoagulation & $2(1.3 \%)$ & $1(1.3 \%))$ \\
DAPT + anticoagulation & $0(0 \%)$ & $0(0 \%)$ \\
$\quad$ Anticoagulation & $2(1.3 \%)$ & $1(0.7 \%)$ \\
Without discharge medication* & $7(4.6 \%)$ & $146(96.7 \%)$ \\
Statins & $141(93.4 \%)$ & $120(79.5 \%)$ \\
Angiotensin-converting enzyme inhibitors/angiotensin-receptor blockers & $107(70.9 \%)$ & 0.185 \\
Beta-blockers & $121(80.1 \%)$ & 0.083 \\
Mineralocorticoid receptor antagonists & $28(18.5 \%)$ & $131(86.8 \%)$ \\
Diuretics & $33(21.9 \%)$ & $19(12.6 \%)$ \\
Calcium blockers & $16(10.6 \%)$ & $37(24.5 \%)$ \\
Nitrates & $100(66.2 \%)$ & 0.122 \\
\hline
\end{tabular}

*In-hospital death $(n=8)$

Table 3. Correlation Between LL-37 Levels and Clinical Variables

\begin{tabular}{lcr}
\hline Variables & Correlation coefficient & $p$ value \\
\hline TG & 0.218 & $<0.001$ \\
TC & 0.154 & 0.008 \\
LDL-C & 0.124 & 0.033 \\
HDL-C & -0.093 & 0.113 \\
TIMI score & -0.209 & $<0.001$ \\
GRACE score & -0.16 & 0.005 \\
\hline
\end{tabular}

Table 4. Cumulative Incidence of Endpoints, Kaplan-Meier Analysis and Multivariable COX Regression Analysis

\begin{tabular}{|c|c|c|c|c|c|c|c|}
\hline & Total & $\begin{array}{l}\text { Low LL-37 } \\
\text { group }\end{array}$ & $\begin{array}{l}\text { High LL-37 } \\
\text { group }\end{array}$ & $\begin{array}{c}\text { Log-rank } \\
p \text { value }\end{array}$ & \multicolumn{3}{|c|}{ Multivariable COX regression* } \\
\hline All-cause death & $24(7.9 \%)$ & $19(12.6 \%)$ & $5(3.3 \%)$ & 0.003 & 0.324 & $0.119-0.879$ & 0.027 \\
\hline Reinfarction & $13(4.5 \%)$ & $10(7.1 \%)$ & $3(2.0 \%)$ & 0.040 & 0.281 & $0.077-1.022$ & 0.054 \\
\hline Unscheduled revascularization & $26(9.1 \%)$ & $18(13.0 \%)$ & $8(5.4 \%)$ & 0.040 & 0.391 & $0.170-0.901$ & 0.027 \\
\hline
\end{tabular}

*Adjusted for age, gender, LVEF, hypertension and diabetes.

(29.1\% vs. $12.6 \%, p=0.0003)$, all-cause death $(12.6 \%$ vs. $3.3 \%, p=0.003)$, reinfarction $(7.1 \%$ vs. $2.0 \%$, $p=0.04)$, and unscheduled revascularization $(13.0 \%$ vs. $5.4 \%, p=0.04)$ were higher in the low than those in the high LL-37 level group. The cumulative incidence of ischemic stroke $(7.0 \%$ vs. $2.7 \%$, $p=0.080)$ was not different between the two groups (Table 4). Kaplan-Meier curves were displayed in Fig. 2 and Fig. 3.
Multivariable Cox regression analysis showed a higher LL-37 level independently predicted lower risk of MACE (hazard ratio [HR] 0.390; 95\% confidence interval [CI] 0.227-0.669; $p<0.001$ ), all-cause death (HR 0.324; 95\%CI 0.119-0.879; $p=0.027$ ), and unscheduled revascularization (HR 0.391; 95\%CI 0.171-0.907; $p=0.027$ ) (Table 4). Subgroup analysis showed the risk reduction associated with higher LL-37 was homogenous across common clinical risk

\section{Advance Publication Journal of Atherosclerosis and Thrombosis}




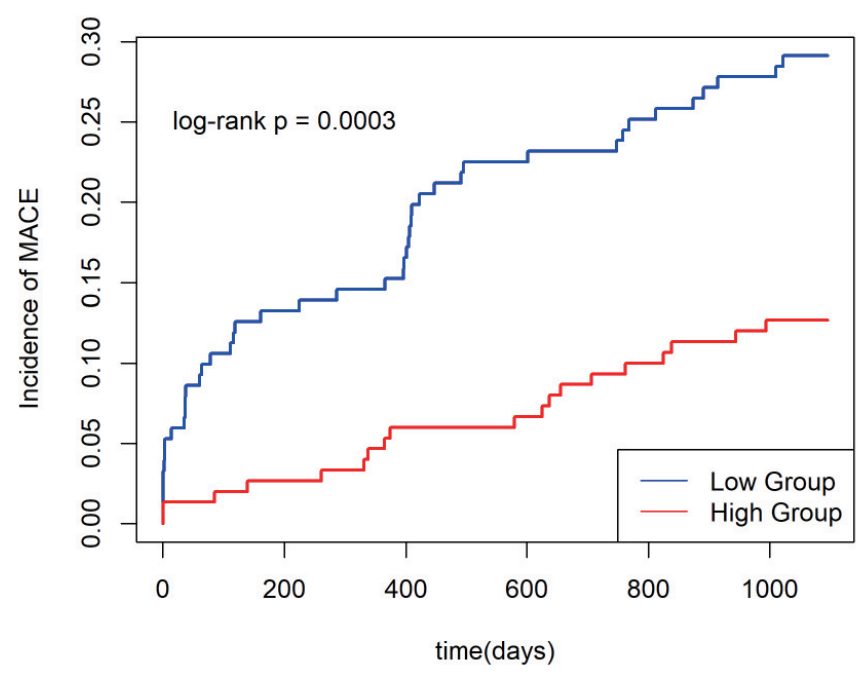

Fig. 2. Kaplan-Meier curves for the cumulative incidence of MACEs, defined as a composite of all-cause mortality, reinfarction, unscheduled revascularization, or ischemic stroke
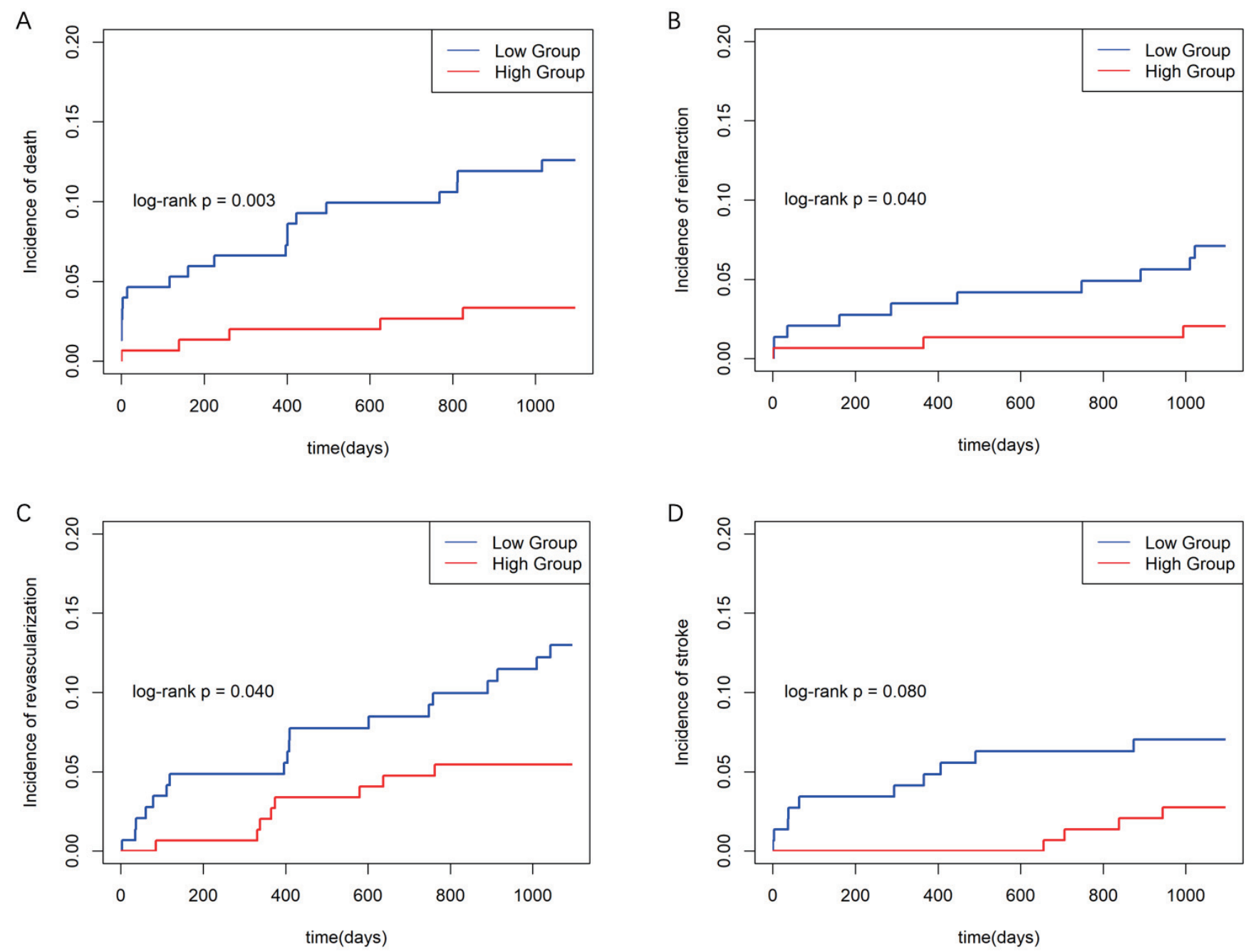

Fig. 3. Kaplan-Meier curves for the cumulative incidence

(A) all-cause mortality; (B) reinfarction; (C) unscheduled revascularization; and (D) ischemic stroke.

Advance Publication Journal of Atherosclerosis and Thrombosis 
Table 5. Subgroup analysis for LL-37 levels and major adverse cardiovascular events by established risk factors

\begin{tabular}{|c|c|c|c|}
\hline Subgroup & $\operatorname{HR}(95 \% \mathrm{CI})$ & $p$-value & $p$ interaction \\
\hline Age $\geq 60$ years & $0.321(0.144-0.713)$ & 0.005 & 0.478 \\
\hline Age $<60$ years & $0.565(0.259-1.233)$ & 0.151 & \\
\hline Male & $0.406(0.215-0.767)$ & 0.006 & 0.973 \\
\hline Female & $0.454(0.156-1.323)$ & 0.148 & \\
\hline $\mathrm{EF} \geq 50 \%$ & $0.477(0.247-0.922)$ & 0.028 & 0.572 \\
\hline $\mathrm{EF}<50 \%$ & $0.314(0.114-0.868)$ & 0.025 & \\
\hline Hypertensive & $0.362(0.188-0.696)$ & 0.002 & 0.534 \\
\hline Non-hypertensive & $0.505(0.184-1.381)$ & 0.183 & \\
\hline Diabetic & $0.718(0.313-1.650)$ & 0.435 & 0.156 \\
\hline Non-diabetic & $0.291(0.137-0.618)$ & 0.001 & \\
\hline $\mathrm{LDL}-\mathrm{C} \geq 2.6 \mathrm{mmol} / \mathrm{L}$ & $0.622(0.301-1.284)$ & 0.199 & 0.086 \\
\hline $\mathrm{LDL}-\mathrm{C}<2.6 \mathrm{mmol} / \mathrm{L}$ & $0.236(0.093-0.597)$ & 0.002 & \\
\hline $\mathrm{TC} \geq 4.1 \mathrm{mmol} / \mathrm{L}$ & $0.505(0.240-1.066)$ & 0.073 & 0.346 \\
\hline $\mathrm{TC}<4.1 \mathrm{mmol} / \mathrm{L}$ & $0.290(0.119-0.707)$ & 0.006 & \\
\hline Triglyceride $\geq 1.7 \mathrm{mmol} / \mathrm{L}$ & $0.703(0.296-1.669)$ & 0.424 & 0.113 \\
\hline Triglyceride $<1.7 \mathrm{mmol} / \mathrm{L}$ & $0.263(0.115-0.601)$ & 0.002 & \\
\hline
\end{tabular}

LVEF: left ventricular ejection fraction; LDL-C: low-density lipoprotein cholesterol; TC: total cholesterol.

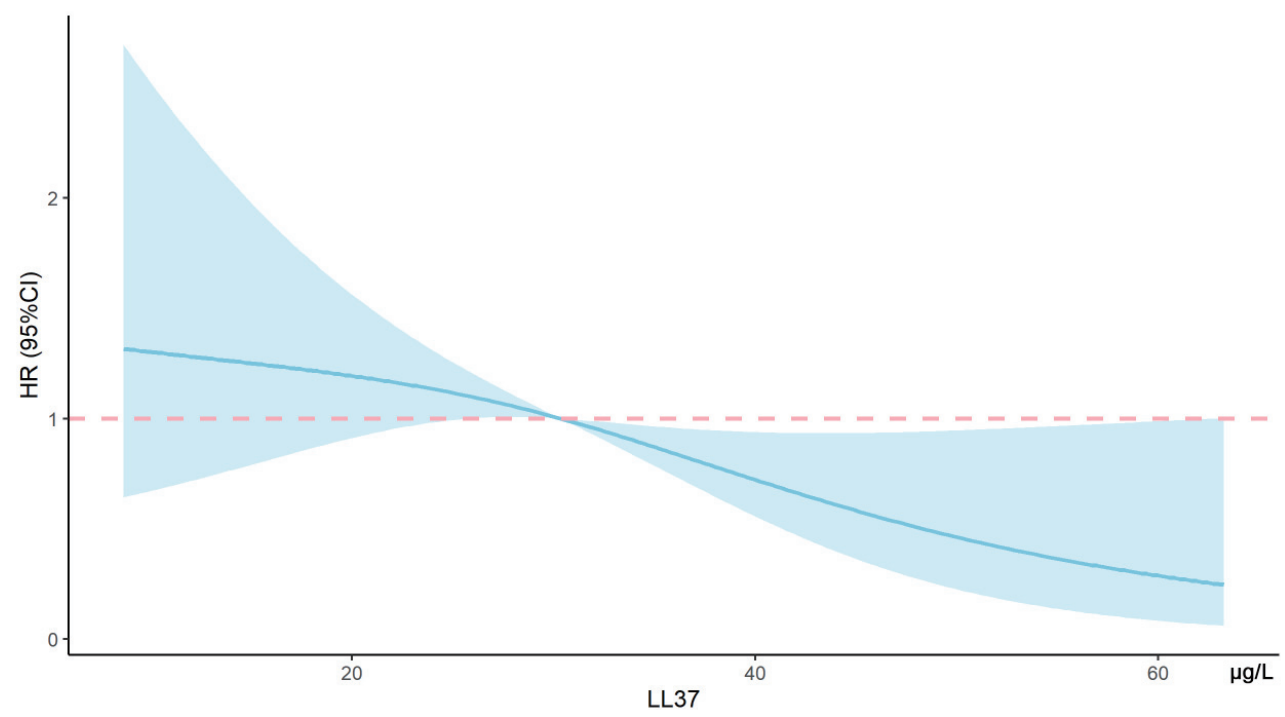

Fig.4. Restricted cubic spline analysis between LL-37 levels and the risk of MACEs

factors (Table 5), including age, gender, LVEF, hypertension, diabetes, levels of LDL-C, TC, and triglycerides. Restricted cubic spline analysis also showed that a higher LL-37 level was constantly associated with a lower risk of MACE (Fig.4), and there were similar results for secondary outcomes (Supplementary Fig. 1). Although with less statistical significance, we associated higher LL-37 levels with decreased risk of reinfarction (HR $0.281 ; 95 \% \mathrm{CI}$ $0.077-1.022 ; p=0.054)$. The LL-37 level was not associated with the risk of ischemic stroke (HR 0.372; 95\%CI 0.116-1.193; $p=0.096$ ) (Table 4).

\section{Discussion}

The major finding of this study is that higher basal plasma levels of human LL-37 may predict decreased 3-year risks of MACE, all-cause death, and unscheduled revascularization in patients after STEMI. In the current study, we showed that lower levels of LL-37 were associated with worse clinical outcomes after STEMI. Two facts supported this finding: 1) the low LL-37 group had higher GRACE and TIMI scores than the high LL-37 group, and LL-37 levels negatively correlated with GRACE and 
TIMI scores. Both of them are established clinical risk scores to predict short- and long-term outcomes after STEMI ${ }^{13-15)}$, and 2) our previous study showed that basal levels of plasma LL-37 were lower in patients with STEMI than those without CAD and stable CAD, and its levels restored within $24 \mathrm{~h}$ after STEMI ${ }^{11)}$. This indicates that decreased blood LL-37 level may be associated with the STEMI pathophysiology or clinical risk.

Although the cumulative incidence of reinfarction was lower in the high than that in the low LL-37 level group $(p=0.04)$, the statistical significance was attenuated after multiple adjustments $(p=0.054)$. This may be due to the relatively small sample size in this study. Comparisons of baseline characteristics revealed that triglyceride, TC, and LDL-C levels were higher in the high than in the low LL-37 group, which positively correlated with LL-37 levels. Unsurprisingly, previous studies showed that triglyceride, TC, and LDL-C levels are lower in the acute phase of AMI, which may predict increased long-term mortality and MACE ${ }^{16-20)}$. Moreover, declined baseline LDL-C level progressively reduces the benefit of intensive lipidlowering therapy in statin-naive patients with acute coronary syndrome ${ }^{17)}$. However, we found no interactions between levels of LL-37 and the above lipid measurements, suggesting that LL-37's protective effect was not altered by dyslipidemia. Our study suggests that LL-37 is probably a factor associated with the residual cardiovascular risk.

LL-37 is a pleotropic bioactive peptide that may not only act as a biomarker for cardiovascular diseases but may also have therapeutic potential. The wellknown protective function of LL-37 is tissue repair. In a randomized, placebo-controlled clinical trial, applying a viscous solution of LL-37 in polyvinyl alcohol with 0.5 to $1.6 \mathrm{mg} / \mathrm{mL}$ concentrations enhanced the healing of hard-to-heal venous leg ulcers compared with the placebo ${ }^{21)}$. This was supported by another clinical study, which showed serum LL-37 concentrations to be strongly associated with healing rates of venous leg ulcers ${ }^{22}$. Patients with poor and moderate wound healing had the lowest serum LL-37 levels, whereas patients with good and fast healing rates had higher LL-37 values ${ }^{22}$. In a mouse model of carotid artery stenting, nitinol stent coated with LL-37 decreased in-stent stenosis ${ }^{9)}$, indicating that LL-37 enhances vascular healing. Further, increased externalization of LL-37 on neutrophil extracellular traps generated from well-controlled type 2 diabetes mellitus neutrophils promoted the wound healing process via fibroblast activation and differentiation using the scratch wound healing assay ${ }^{23)}$. An in vitro study showed that LL-37 inhibited the adenosine diphosphate, collagen, and U46619 (11,9 epoxymethano-prostaglandin $\mathrm{H} 2$, a selective agonist of prostaglandin $\mathrm{H} 2 /$ thromboxane $\mathrm{A} 2$ receptor) induced human platelet aggregation in a dosedependent manner ${ }^{10)}$. LL-37 also inhibited P-selectin expression on human platelets, decreased human platelet spreading on immobilized fibrinogen, and attenuated arteriovenous shunt thrombosis in rats ${ }^{10)}$. These results demonstrated that LL-37 has antiplatelet and antithrombotic functions. LL-37 at $0.5-5 \mu \mathrm{M}$ range also increases endothelial cell stiffness of various cell types. It reduces endothelial cell permeability coincident with the remodeling of the actin cytoskeleton and adherens junction proteins, indicating that LL-37 may enforce endothelial barrier integrity $^{24)}$. These findings suggest that human LL-37 may have beneficial effects on the cardiovascular system.

Although LL-37's antimicrobial and immunomodulatory functions have been intensively investigated, the mechanisms of its potential protective roles on atherosclerosis and AMI are widely unknown. LL-37 has $46 \%$ identity in amino acid sequence with the mouse cathelicidin-related antimicrobial peptide (CRAMP). Thus, CRAMP mechanisms involved in AMI may provide some information about LL-37. Recent studies have shown that CRAMP has protective roles in mouse AMI models. In the mouse myocardial ischemia/reperfusion injury model, intraperitoneal injection of CRAMP peptide 3 days before ligation of the left anterior descending artery reduced cardiomyocyte apoptosis and myocardial ischemia/reperfusion injury ${ }^{25)}$. In contrast, CRAMP knockdown enhances cardiomyocyte apoptosis, whereas CRAMP knockout (CRAMP-/-) increases the infarct size and myocardial apoptosis. This may be mediated by activating Akt and ERK1/2 and phosphorylation and nuclear export of FoxO3a using CRAMP ${ }^{25)}$. Moreover, injection of bone-marrowderived stem/progenitor cells (BMSPCs) preincubated with CRAMP around the infarct border zones enhanced cardiac recovery and reduced the scar size and adverse remodeling ${ }^{26}$. Similarly, sustained CRAMP release from hydrogels injected into the infarction border zone enhanced the functional recovery, increased capillary density, and decreased scar size. This effect may be attributable to enhanced BMSPC retention/recruitment and the therapeutic effect of stromal-derived factor ${ }^{26)}$.

However, some studies demonstrated that CRAMP or LL-37 might be prothrombotic or proatherosclerotic. In a carotid artery ligation model, transplanting the bone marrow of CRAMP-/- mice to wild-type $(\mathrm{CRAMP}+/+)$ mice reduced thrombus 
formation, maximal thrombus size, and duration of vessel occlusion, diminished platelet adhesion, and aggregate formation ${ }^{27)}$. This suggests that hematopoietic CRAMP contributes to arterial thrombus formation and stability. Compared with ApoE-/- mice, CRAMP-/-ApoE-/- mice had a smaller atherosclerotic lesion size with lower macrophage numbers after 4 weeks of high-fat diet ${ }^{28)}$. In a cell perfusion model over tumor necrosis factor (TNF)-activated monolayers of human aortic endothelial cells, deposition $1 \mu \mathrm{g}$ of LL-37 to the endothelial medium 15 min before perfusion induced adhesion of classical monocytes and neutrophils; however, nonclassical monocytes were not affected ${ }^{28)}$. This suggests that LL-37 may enhance the recruitment of inflammatory monocytes. An LL-37 and mitochondrial DNA (mtDNA) complex appeared in the human plasma and atherosclerotic lesions. This complex induced the release of interleukin-6 (IL-6), IL-8, TNF- $\alpha$, and matrix metalloproteinase 8 in purified polymorphonuclear leukocyte (PMNs), indicating that the LL-37-mtDNA complex may activate $\mathrm{PMNs}^{29)}$. LL-37-mtDNA stimulation on HUVECs triggered Toll-like receptor 9 (TLR9)mediated secretion of chemokines and cytokines, including monocyte chemotactic protein-1, intercellular cell adhesion molecule-1, and IL-8 ${ }^{29)}$. Injection of CRAMP-mtDNA complex into ApoE-/mice $(120 \mathrm{mg} /$ mouse, 3 times per week for 4 weeks) at a high-fat diet increased the atherosclerotic lesion in the aortic root. In contrast, injection of anti-CRAMPmtDNA complex antibody $(50 \mathrm{mg} /$ mouse, twice a week for 5 weeks) reduced the lesion size. These findings suggest that the LL-37-mtDNA complex may act as a mediator of atherosclerosis formation ${ }^{29)}$.

The percentage identity in amino acid sequence between the LL-37 and CRAMP is 46\%, which is considerably lower than the average degree of protein sequence identity $(85 \%)$ between the orthologs of mouse and human genes ${ }^{30)}$. Thus, it is not conclusive that we could consider mouse CRAMP as the ortholog of human LL-37. Furthermore, some studies have shown that these two molecules have different activities. For example, LL-37 could bind doublestranded ribonucleic acid (dsRNA) and upregulate TLR3 signaling by trafficking endosomes, where TLR3 resides. CRAMP does not bind dsRNA and cannot activate TLR3 signaling. Thus, we should cautiously apply the findings of CRMAP to LL-37. A clinical study indicated that LL-37 overdose might be ineffective or harmful. For example, topical application of 0.5 or $1.6 \mathrm{mg} / \mathrm{mL}$ of human recombinant LL-37 enhances the healing of venous leg ulcers. However, a $3.2 \mathrm{mg} / \mathrm{mL}$ dose is less effective and associated with an increased risk of adverse reaction (ulcer necrosis or severe inflammatory reaction $)^{21)}$. Thus, experimental and clinical studies investigating the effects of LL-37 on atherosclerotic diseases should investigate the optimum dose and inquiry if it is attributable to the toxic effect of overdosing when harmful results occur.

There are some limitations to the current study. First, the study was performed in a single-center, and the number of patients was relatively small. Second, patients with cardiogenic shock were excluded. These may have led to selection bias. Therefore, a future multi-center, large-scale study involving cardiogenic shock patients is expected to confirm the present study's findings.

\section{Conclusion}

In conclusion, a higher basal plasma LL-37 level may predict decreased 3-year risks of ischemic events after STEMI. Together with the findings of previous studies $^{11,25,26)}$, our results indicate that LL-37 might be protective in atherosclerotic diseases. The LL-37 peptide or options enhancing native LL-37 production may have therapeutic potential for patients with AMI.

\section{Funding Source}

This study was supported by Chinese Academy of Medical Sciences Innovation Fund for Medical Sciences (No.2016-I2M-1-009), Fundamental Research Funds for Central Public-interest Scientific Institution (2012-F25), Fund of "Sanming" Project of Medicine in Shenzhen (No.SZSM201911017) and Shenzhen Key Medical Discipline Construction Fund (No.SZXK001).

\section{Conflicts of Interest Statement}

The authors declare that there is no conflict of interest.

\section{References}

1) Virani SS, Alonso A, Benjamin EJ, Bittencourt MS, Callaway CW, Carson AP, Chamberlain AM, Chang AR, Cheng S, Delling FN, Djousse L, Elkind MSV, Ferguson JF, Fornage M, Khan SS, Kissela BM, Knutson KL, Kwan TW, Lackland DT, Lewis TT, Lichtman JH, Longenecker CT, Loop MS, Lutsey PL, Martin SS, Matsushita K, Moran AE, Mussolino ME, Perak AM, Rosamond WD, Roth GA, Sampson UKA, Satou GM, Schroeder EB, Shah SH, Shay CM, Spartano NL, Stokes A, Tirschwell DL, VanWagner LB, Tsao CW, American Heart Association Council on E, Prevention Statistics C, Stroke Statistics S. Heart Disease and Stroke Statistics-2020 
Update: A Report From the American Heart Association. Circulation, 2020; 141: e139-e596

2) Libby P, Okamoto $Y$, Rocha VZ, Folco E. Inflammation in atherosclerosis: transition from theory to practice. Circ J, 2010; 74: 213-220

3) Wolf D, Ley K. Immunity and Inflammation in Atherosclerosis. Circ Res, 2019; 124: 315-327

4) Gistera A, Hansson GK. The immunology of atherosclerosis. Nat Rev Nephrol, 2017; 13: 368-380

5) Sorensen OE, Follin P, Johnsen AH, Calafat J, Tjabringa GS, Hiemstra PS, Borregaard N. Human cathelicidin, hCAP-18, is processed to the antimicrobial peptide LL-37 by extracellular cleavage with proteinase 3 . Blood, 2001; 97: 3951-3959

6) Bandurska K, Berdowska A, Barczynska-Felusiak R, Krupa P. Unique features of human cathelicidin LL-37. Biofactors, 2015; 41: 289-300

7) Durr UH, Sudheendra US, Ramamoorthy A. LL-37, the only human member of the cathelicidin family of antimicrobial peptides. Biochim Biophys Acta, 2006; 1758: $1408-1425$

8) Vandamme D, Landuyt B, Luyten W, Schoofs L. A comprehensive summary of LL-37, the factotum human cathelicidin peptide. Cell Immunol, 2012; 280: 22-35

9) Soehnlein O, Wantha S, Simsekyilmaz S, Doring Y, Megens RT, Mause SF, Drechsler M, Smeets R, Weinandy S, Schreiber F, Gries T, Jockenhoevel S, Moller M, Vijayan S, van Zandvoort MA, Agerberth B, Pham CT, Gallo RL, Hackeng TM, Liehn EA, Zernecke A, Klee D, Weber C. Neutrophil-derived cathelicidin protects from neointimal hyperplasia. Sci Transl Med, 2011; 3: 103ra198

10) Su W, Chen Y, Wang C, Ding X, Rwibasira G, Kong Y. Human cathelicidin LL-37 inhibits platelet aggregation and thrombosis via Src/PI3K/Akt signaling. Biochem Biophys Res Commun, 2016; 473: 283-289

11) Zhao H, Yan H, Yamashita S, Li W, Liu C, Chen Y, Zhou P, Chi Y, Wang S, Zhao B, Song L. Acute ST-segment elevation myocardial infarction is associated with decreased human antimicrobial peptide LL-37 and increased human neutrophil peptide-1 to 3 in plasma. J Atheroscler Thromb, 2012; 19: 357-368

12) Ibanez B, James S, Agewall S, Antunes MJ, BucciarelliDucci C, Bueno H, Caforio ALP, Crea F, Goudevenos JA, Halvorsen S, Hindricks G, Kastrati A, Lenzen MJ, Prescott E, Roffi M, Valgimigli M, Varenhorst C, Vranckx P, Widimsky P, Group ESCSD. 2017 ESC Guidelines for the management of acute myocardial infarction in patients presenting with ST-segment elevation: The Task Force for the management of acute myocardial infarction in patients presenting with ST-segment elevation of the European Society of Cardiology (ESC). Eur Heart J, 2018; 39: 119-177

13) Morrow DA, Antman EM, Charlesworth A, Cairns R, Murphy SA, de Lemos JA, Giugliano RP, McCabe $\mathrm{CH}$, Braunwald E. TIMI risk score for ST-elevation myocardial infarction: A convenient, bedside, clinical score for risk assessment at presentation: An intravenous nPA for treatment of infarcting myocardium early II trial substudy. Circulation, 2000; 102: 2031-2037

14) Fox KA, Dabbous OH, Goldberg RJ, Pieper KS, Eagle KA, Van de Werf F, Avezum A, Goodman SG, Flather
MD, Anderson FA, Jr., Granger CB. Prediction of risk of death and myocardial infarction in the six months after presentation with acute coronary syndrome: prospective multinational observational study (GRACE). BMJ, 2006; 333: 1091

15) Kozieradzka A, Kaminski KA, Maciorkowska D, Olszewska M, Dobrzycki S, Nowak K, Kralisz P, Prokopczuk P, Musial WJ. GRACE, TIMI, Zwolle and CADILLAC risk scores--do they predict 5-year outcomes after ST-elevation myocardial infarction treated invasively? Int J Cardiol, 2011; 148: 70-75

16) Jackson R, Scragg R, Marshall R, White H, O'Brien K, Small C. Changes in serum lipid concentrations during first 24 hours after myocardial infarction. Br Med J (Clin Res Ed), 1987; 294: 1588-1589

17) Giraldez RR, Giugliano RP, Mohanavelu S, Murphy SA, McCabe CH, Cannon CP, Braunwald E. Baseline lowdensity lipoprotein cholesterol is an important predictor of the benefit of intensive lipid-lowering therapy: a PROVE IT-TIMI 22 (Pravastatin or Atorvastatin Evaluation and Infection Therapy-Thrombolysis In Myocardial Infarction 22) analysis. J Am Coll Cardiol, 2008; 52: 914-920

18) Cheng YT, Liu TJ, Lai HC, Lee WL, Ho HY, Su CS, Liu $\mathrm{CN}$, Wang KY. Lower serum triglyceride level is a risk factor for in-hospital and late major adverse events in patients with ST-segment elevation myocardial infarction treated with primary percutaneous coronary interventiona cohort study. BMC Cardiovasc Disord, 2014; 14: 143

19) Khawaja OA, Hatahet H, Cavalcante J, Khanal S, Al-Mallah MH. Low admission triglyceride and mortality in acute coronary syndrome patients. Cardiol J, 2011; 18(3): 297-303

20) Al-Mallah MH, Hatahet $H$, Cavalcante JL, Khanal S. Low admission LDL-cholesterol is associated with increased 3-year all-cause mortality in patients with non ST segment elevation myocardial infarction. Cardiol J, 2009; 16: 227-233

21) Gronberg A, Mahlapuu M, Stahle M, Whately-Smith C, Rollman O. Treatment with LL-37 is safe and effective in enhancing healing of hard-to-heal venous leg ulcers: a randomized, placebo-controlled clinical trial. Wound Repair Regen, 2014; 22: 613-621

22) Krejner A, Litwiniuk M, Grzela T. LL-37 but Not 25-Hydroxy-Vitamin D Serum Level Correlates with Healing of Venous Leg Ulcers. Arch Immunol Ther Exp (Warsz), 2017; 65: 455-461

23) Arampatzioglou A, Papazoglou D, Konstantinidis T, Chrysanthopoulou A, Mitsios A, Angelidou I, Maroulakou I, Ritis K, Skendros P. Clarithromycin Enhances the Antibacterial Activity and Wound Healing Capacity in Type 2 Diabetes Mellitus by Increasing LL-37 Load on Neutrophil Extracellular Traps. Front Immunol, 2018; 9: 2064

24) Byfield FJ, Wen Q, Leszczynska K, Kulakowska A, Namiot Z, Janmey PA, Bucki R. Cathelicidin LL-37 peptide regulates endothelial cell stiffness and endothelial barrier permeability. Am J Physiol Cell Physiol, 2011; 300: C105-112

25) Bei Y, Pan LL, Zhou Q, Zhao C, Xie Y, Wu C, Meng X, Gu H, Xu J, Zhou L, Sluijter JPG, Das S, Agerberth B, 
Sun J, Xiao J. Cathelicidin-related antimicrobial peptide protects against myocardial ischemia/reperfusion injury. BMC Med, 2019; 17: 42

26) Klyachkin YM, Idris A, Rodell CB, Tripathi H, Ye S, Nagareddy P, Asfour A, Gao E, Annabathula R, Ratajczak M, Burdick JA, Abdel-Latif A. Cathelicidin Related Antimicrobial Peptide (CRAMP) Enhances Bone Marrow Cell Retention and Attenuates Cardiac Dysfunction in a Mouse Model of Myocardial Infarction. Stem Cell Rev Rep, 2018; 14: 702-714

27) Pircher J, Czermak T, Ehrlich A, Eberle C, Gaitzsch E, Margraf A, Grommes J, Saha P, Titova A, IshikawaAnkerhold H, Stark K, Petzold T, Stocker T, Weckbach LT, Novotny J, Sperandio M, Nieswandt B, Smith A, Mannell H, Walzog B, Horst D, Soehnlein O, Massberg $S$, Schulz C. Cathelicidins prime platelets to mediate arterial thrombosis and tissue inflammation. Nat Commun, 2018; 9: 1523

28) Doring Y, Drechsler M, Wantha S, Kemmerich K, Lievens D, Vijayan S, Gallo RL, Weber C, Soehnlein O. Lack of neutrophil-derived CRAMP reduces atherosclerosis in mice. Circ Res, 2012; 110: 1052-1056

29) Zhang Z, Meng P, Han Y, Shen C, Li B, Hakim MA, Zhang X, Lu Q, Rong M, Lai R. Mitochondrial DNA-LL-37 Complex Promotes Atherosclerosis by Escaping from Autophagic Recognition. Immunity, 2015; 43: 1137-1147

30) Makalowski W, Zhang J, Boguski MS. Comparative analysis of 1196 orthologous mouse and human fulllength mRNA and protein sequences. Genome Res, 1996; 6: 846-857 
A

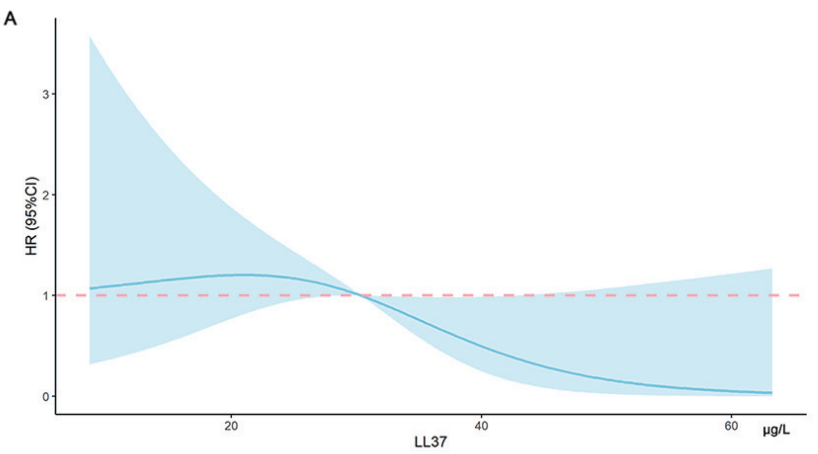

c

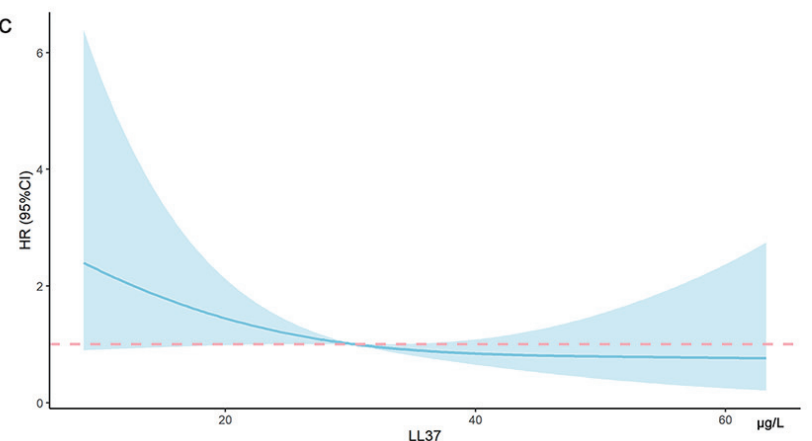

B

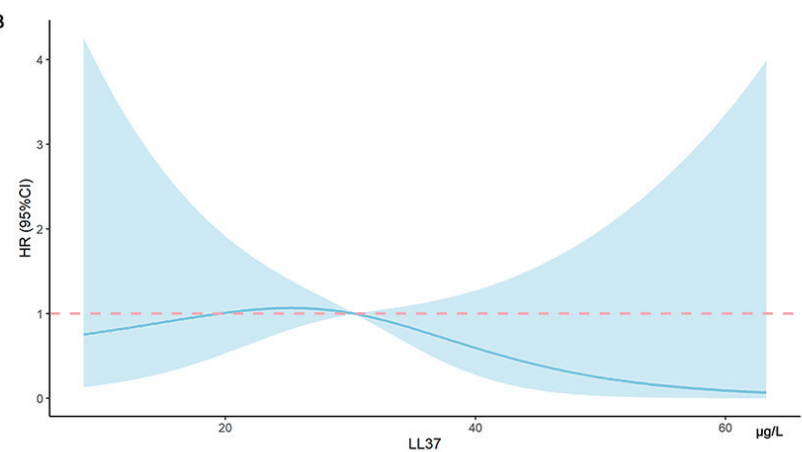

D

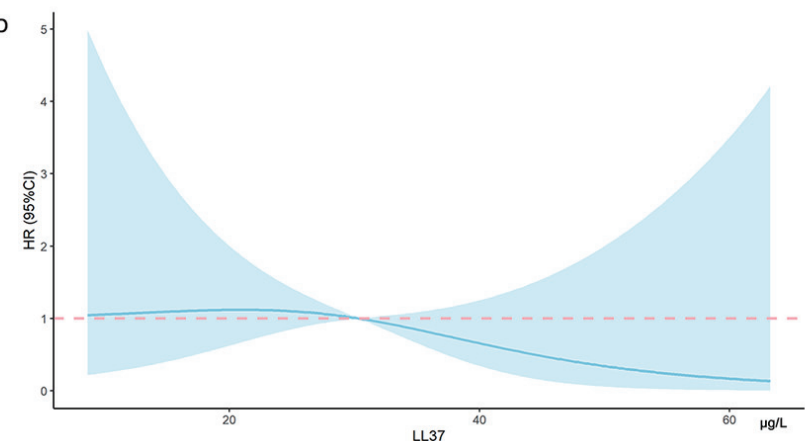

Supplementary Fig. 1. Restricted cubic spline analysis between LL-37 levels and the risks of all-cause mortality (A), reinfarction (B), unscheduled revascularization $(\mathrm{C})$, and ischemic stroke (D) 